\title{
LONGITUDINAL FOLLOW-UP OF CREATINE KINASE AND MARKERS OF RENAL FUNCTION IN MALE BASKETBALL PLAYERS
}

\author{
Anatoli Landõr ${ }^{1}$, Algirdas Juozulynas ${ }^{2}$, Zhanar Andasova ${ }^{3}$,

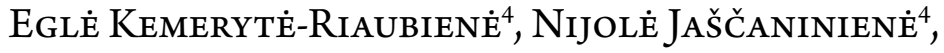 \\ AUdRIUS GOCENTAS ${ }^{2,4}$ \\ ${ }^{1}$ Faculty of Medicine, University of Tartu, Estonia \\ ${ }^{2}$ State Research Institute Centre for Innovative Medicine, Lithuania \\ ${ }^{3}$ Kazakh Academy of Sport and Tourism, Kazakhstan \\ ${ }^{4}$ Lithuanian University of Educational Sciences, Lithuania
}

\begin{abstract}
The results from different sports studies regarding changes in parameters of renal function as a consequence of exercise-induced muscular damage are conflicting.

Objective: The aim of this study was to investigate the dynamics of renal function in association with the phases of the macro cycle and to establish possible relationship between indicators of muscle damage and renal function in basketball players.

Methods: Nine professional basketball players were monitored during 12 weeks starting from the first day of pre-season preparations. Blood samples were collected three times: before the pre-season, during the pre-season and during the in-season to determine blood creatine kinase (CK), creatinine and urea concentrations and to estimate glomerular filtration rate (eGFR).

Results: The ANOVA test revealed statistically significant changes in CK and eGFR, associated with physical loads in the basketball players. However, the test did not confirm relationships between indicators of renal function and muscle damage during any phase of the macro cycle.

Conclusion: Elevated levels of CK induced by sport-specific activities are not associated with changes in renal function despite the use of frequent and intense physical loads in male professional basketball players. Further research is necessary to elucidate regularities in changes of renal function among team sport representatives.
\end{abstract}

Keywords: monitoring, renal function, muscle damage, basketball 


\section{INTRODUCTION}

Intense training and the growing number of competitions are attributes of contemporary-professional sport $[11,16,17]$. Adequate monitoring of the functional status is necessary to ensure the quality of recovery and hence further successful participation in professional athletic activities [18]. Monitoring of recovery is complicated because of the absence of simple and valuable indicators. It is mainly conducted by detection of normalisation of biochemical and other variables previously disturbed by exercising or competing. The wide choice of metabolic markers in sports medicine was recently systematised by Banfi and colleagues [2]. Among others, indicators of muscle damage such as creatine kinase (CK) and myoglobin have been studied extensively and applied practically as valid markers of muscle micro trauma [4]. Muscle damage after physical effort can be associated with changes in renal function, namely with elevation of creatinine levels and decrease of glomerular filtration rate [7]. Serum creatinine level has become the most commonly used measure of kidney function in the last four decades. Assessment of renal function is an obligatory part of the evaluation of an athlete's general health. Despite the absence of specialised normative ranges for sportsmen, relationships between creatinine level and morphological properties have been described $[1-3,9,13,20]$. The significance of the relationship between muscle damage and changes in parameters of renal function has been confirmed mostly in endurance sports $[7,10]$ and in studies focusing on experimental protocols of exercise [15]. Eccentric training associated with CK elevation above $10000 \mathrm{U} / 1$ was not associated with renal pathology in a study of Clarkson et al. [6]. Meyer and Meister [19] described significant changes in creatinine concentrations in a large cohort of professional soccer players and suggested that supplementing of creatine could induce such findings. Another study suggests that anthropometric properties as well as methods of analysis have influenced indices of renal function in rugby players [9]. Basketball players were involved in only one comparative study among other seven sports representatives, which confirmed direct correlation between body mass index and creatinine levels [1]. There is lack of data regarding the dynamics of renal function in basketball. As shown in our previous research, intense training and frequent matches were associated with prolonged stress and muscle damage in basketball players [8]. We suggested that a long competitive season involving frequent matches and intense training could influence renal function, as a consequence of applying repeated physical loads, through 
chronically stimulating CK activity. The aim of this study was to investigate the dynamics of renal function in association with the phases of the macro cycle and to establish possible relationships between muscle damage, creatinine level and glomerular filtration rate.

\section{MATERIAL AND METHODS}

Nine professional basketball players, aged $23.7 \pm 3$ years, weight $96.9 \pm 9.27 \mathrm{~kg}$, height $199.7 \pm 8.3 \mathrm{~cm}$, maximal oxygen consumption $49.5 \pm 3.86 \mathrm{ml} \cdot \mathrm{kg}^{-1} \cdot \mathrm{min}^{-1}$, average levels of haemoglobin $152.1 \pm 9.73 \mathrm{~g} / \mathrm{dL}$ at the beginning of the observation, were included in this study.

All subjects followed the same structured training programme which was comparable with the programmes described by Manzi et al. [16] and included training sessions, matches and rest days. Basketball drills, tactics, sprints, intermittent running exercises, and specific conditioning activity, as well as weight training and plyometrics served as the components of the training sessions of the involved athletes.

The athletes took part in the Lithuanian Basketball League, in the Baltic Championship and in the Euroleague. Each athlete gave written informed consent before the start of the study. The study design and the procedures used were in accordance with ethical standards and with the Declaration of Helsinki. All subjects followed a similar diet and did not receive special supplements, except for a complex of multivitamins and minerals throughout the season.

The study procedure was adapted to the annual macro cycle of the athletes. Measurement of biochemical variables was conducted three times during the characteristic training phases of the annual cycle: the pre-season, the in-season, and the off-season. Three examinations were carried out at the time points representing the above phases of the yearly cycle. The first examination represents the status of the players during the off-season and was done before the first team practice in the pre-season in mid-August. The second examination was done after the fifth week of pre-season training in the second half of September. The third blood sampling representing the first half of the in-season was done at the end of October.

To avoid any confounding effects of variations in circadian rhythm and food intake, the athletes provided blood samples at the same time (09.00-09.30) after overnight fasting. The samplings were done $14 \mathrm{~h}$ after the last physical 
load, except for the first sample. All venous blood samples were drawn via antecubital venipuncture in a semirecumbent position. Blood was collected into Vacutainer tubes. Analysed parameters were determined by standard clinical laboratory techniques. Serum creatine kinase (CK) activity was assessed using the IFCC method and an Advia 1650 analyser (Siemens Healthcare Diagnostics, Deerfield, IL, USA). Serum levels of urea and creatinine were measured using an automatic autoanalyser Advia 1650. Estimated glomerular filtration rate (eGFR) was obtained using the Modification of Diet in Renal Disease (MDRD) equation [12].

The Kolmogorov-Smirnov test was used to check the normality of the data. One-way ANOVA with repeated measures was used to evaluate differences in CK, creatinine, urea and eGFR across the three time points. Tukey's HSD post hoc test was performed to assess the significance of difference between consecutive examinations. Significance level was set at $\mathrm{p} \leq 0.05$.

\section{RESULTS}

The Kolmogorov - Smirnov test revealed a normal distribution of the analysed variables. The results of the ANOVA test as well as the values of the investigated variables are presented in Table 1.

Table 1. Comparative values of the analysed variables of muscle damage and renal function during different periods of the macro cycle in male professional basketball players.

\begin{tabular}{lccccc}
\hline \multicolumn{1}{c}{ Parameters } & Off-season & Pre-season & In-season & $\mathbf{F}$ & $\mathbf{p}$ \\
\hline Creatinine, $\mu \mathrm{mol} / \mathrm{I}$ & $83.6 \pm 13.67$ & $90.9 \pm 8.16$ & $96.3 \pm 9.51$ & 3.2 & 0.057 \\
\hline $\mathrm{CK}, \mathrm{U} / \mathrm{l}$ & $158.1 \pm 134.08$ & $366.1 \pm 173.07$ & $462.7 \pm 257.83$ & 5.7 & 0.009 \\
\hline Urea, $\mathrm{mmol} / \mathrm{l}$ & $6.8 \pm 1.65$ & $7.2 \pm 1.81$ & $7.0 \pm 1.63$ & 0.4 & 0.864 \\
\hline eGFR, $\mathrm{ml} / \mathrm{min} / 1.73 \mathrm{~m}^{2}$ & $108.3 \pm 23.73$ & $95.7 \pm 8.94$ & $89.5 \pm 9.3$ & 3.4 & 0.05 \\
\hline
\end{tabular}

The results of ANOVA in Table 1 revealed a significant increase in CK levels. Post hoc analysis using the Tukey HSD criterion confirmed the difference in the $\mathrm{CK}$ values between the off-season and the in-season ( $\mathrm{p}=0.008)$. Our results suggest some trend towards negative changes in the parameters of renal function except for the urea levels after the beginning of structured training. The post hoc analysis, using the Tukey HSD criterion, confirmed the difference in eGFR changes between the off-season and the in-season $(p=0.044)$. 
Graphically, the relationships between the parameters of renal function and $\mathrm{CK}$ in different periods of the macro cycle are shown in Figures 1 and 2. The first graph presents the relationship between muscle damage and creatinine levels in the professional basketball players.

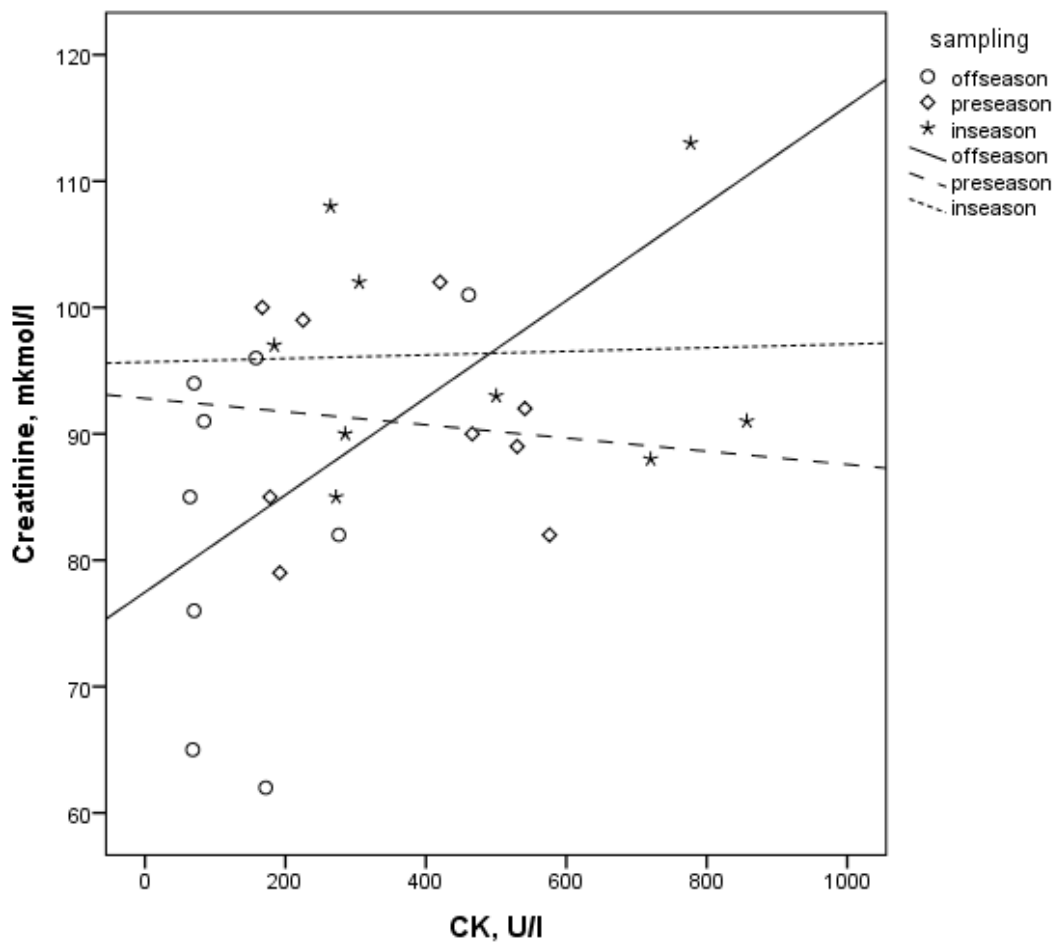

Figure 1. The scatterplots of individual values of $\mathrm{CK}$ activity and creatinine depending on the phase of the macro cycle.

In all phases of observation, correlation coefficient $r$ was less than 0.4 and a $p$ value exceeded 0.05 .

The estimated GFR data were included in the second graph in search for correlation with CK levels in different periods of the macro cycle. 


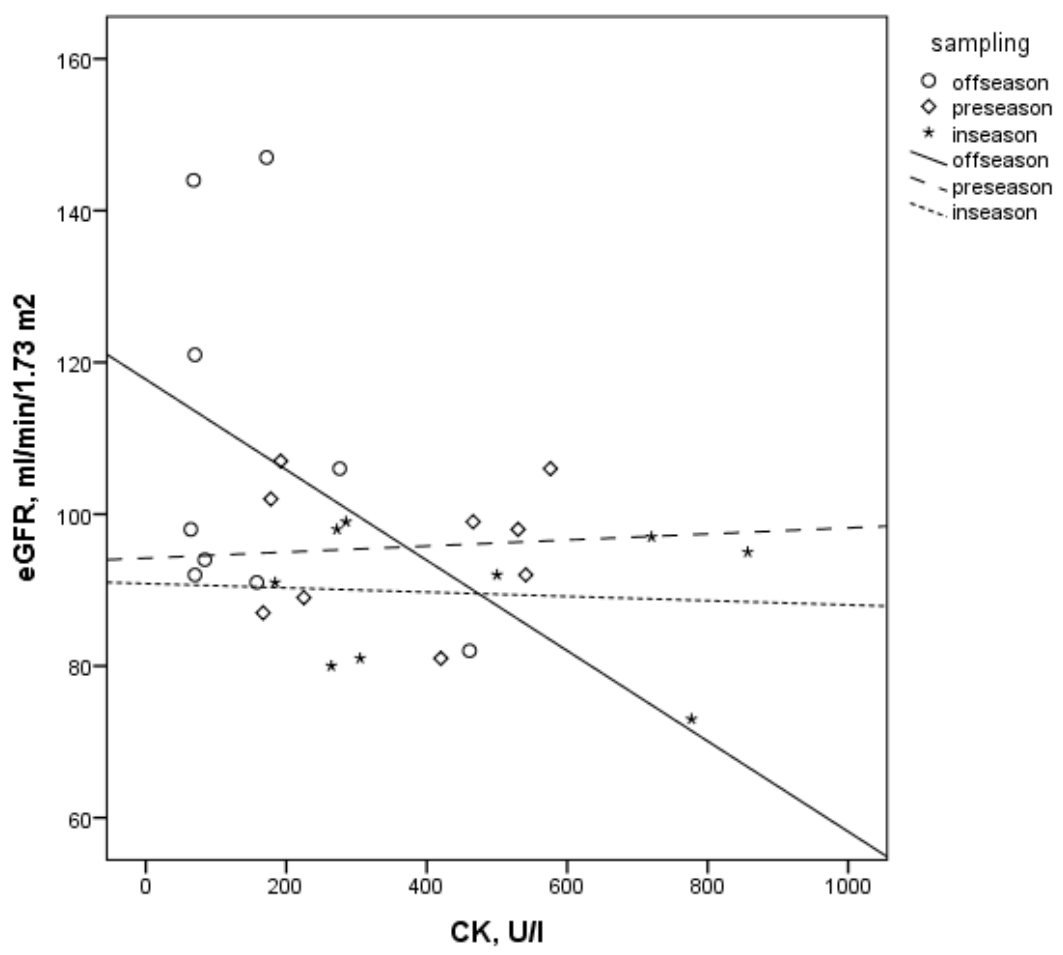

Figure 2. The scatterplots of individual values of CK activity and estimated GFR depending on the phase of the macro cycle.

There were some statistically significant correlations between CK and eGFR as shown in Figure 2 ( $p>0.05)$.

\section{DISCUSSION}

The aim of this study was to follow the course of renal function across the main points of the yearly macro cycle and to investigate possible association between muscle damage and renal function in male professional basketball players. The detected increase of CK levels associated with structured training activities after the off-season is expected and is consistent with the data from other team sports studies [9, 11, and 17]. Also, we found significant changes in eGFR depending on the phases of the macro cycle, however, the clinical relevance of this finding remains unclear primarily because of a very narrow variation which fits the normative range. Estimation of glomerular filtration rate depends on the 
equation used $[1-3,7,13,20]$. We opted to use the MDRD equation because among other equations it yields the strongest correlation with CK activity [15]. Another reason was that the MDRD equation is not sensitive to body mass or body mass index, which have an impact on the result of eGFR $[1,9,13,20]$.

Confirmation of the detected trends in the parameters of renal function probably requires a longer period of observation. Nevertheless, our results failed to confirm any relationship between muscle damage and changes in creatinine levels or estimated glomerular filtration rate in the male basketball players. The design of our study is similar to that of a study by Reinke et al. [21]. They investigated the levels of creatinine at the same time points of the macro cycle, namely the off-season, the pre-season and the in-season, and concluded that the highest levels of creatinine during the off-season in soccer players were associated with an increased rate of muscle turnover. Our data do not confirm this trend but rather the opposite as lower creatinine values were observed namely in the off-season, as shown in Table 1.

Creatine intake, quality of hydration and renal diseases were regarded as the most probable reasons influencing changes in creatinine level among professional soccer players [19]. Despite the prevalent opinion that creatine intake evokes the rise of creatinine levels, Cancela et al. [5] noted the absence of impact on creatinine levels after supplementing $8 \mathrm{~g}$ per day of creatine in soccer players. A similar conclusion was made by Lugaresi et al. [14] after supplementing creatine for 12 weeks in resistance-trained subjects. Although we cannot exclude the possibility that our results were affected by creatine supplementation, the involved athletes disclaimed additional individual supplementation throughout the period of observation. Nor could the quality of hydration have influenced the results of the current study as the athletes could drink ad libitum during breaks and after practices and competitions. Renal diseases were excluded during a medical check-up in the pre-season. We can suggest that the quality of recovery could have influenced our findings, especially when keeping in mind shorter than recommended [17] rest periods between consecutive physical loads. Previous data about confirmed correlations between muscle damage and changes of renal function pertained to representatives of endurance sports or resistance-trained subjects $[7,10,15]$. Despite the different nature of physical loads in the above studies, they can be described as requiring ultimate exertion, incomparable to loads used in team sport games. The absence of relationship between $\mathrm{CK}$ and renal function in our study probably suggests that the 
degree of muscular damage sustained during basketball-specific activities is, despite their high intensity and frequency, too low to evoke clinically relevant changes of renal function.

Our aim to search for possible relationship between muscle damage and renal function in high-level basketball players in the setting of the training process was prompted by practical considerations. Previous research of team sport games suggests very high and (too?) frequent physical loads in current soccer, rugby and basketball professional players $[11,16,17]$. Manzi et al. [16] described a typical micro cycle in professional basketball with two matches per week. The weekly schedule of the investigated athletes included 2-3 matches, which implies the absence of rest for $48 \mathrm{~h}$, as suggested by McLellan et al. [17], and possible under-recovery during the in-season.

This study has several limitations. Differentiation between the impacts of general training and last training is an unresolved issue in athletic monitoring studies. We can only speculate that the rise in creatinine levels is cumulative in nature, while the opposite standpoint, i.e. that the obtained values demonstrate the impact of the last training session or competition, are open to discussion. Also, the number of the study subjects is small. Hence, the obtained data should be confirmed by more representative studies with longer follow-up.

In conclusion, the hypercreatinekinasaemia induced by basketball-specific activities is not associated with changes in renal function despite application of frequent and intense physical loads in male professional players. Further research is necessary to elucidate regularities in changes of renal function among team sport representatives.

\section{REFERENCES}

1. Banfi G., Del Fabbro M., Lippi G. (2006). Relation between serum creatinine and body mass index in elite athletes of different sport disciplines. Br J Sports Med, 40(8), 675-8.

2. Banfi G., Colombini A., Lombardi G., et al. (2012). Metabolic markers in sports medicine. Adv Clin Chem, 56, 1-54.

3. Banfi G., Del Fabbro M., Lippi G. (2009). Serum creatinine concentration and creatinine-based estimation of glomerular filtration rate in athletes. Sports Med, 39(4), 331-7.

4. Brancaccio P., Maffulli N., Limongelli F. M. (2007). Creatine kinase monitoring in sport medicine. Br Med Bull, 81-82, 209-30. 
5. Cancela P., Ohanian C., Cuitiño E., et al. (2008). Creatine supplementation does not affect clinical health markers in football players. Br J Sports Med, 42(9), 731-5.

6. Clarkson P. M., Kearns A. K., Rouzier P. et al. (2006). Serum creatine kinase levels and renal function measures in exertional muscle damage. Med Sci Sports Exerc, 38(4), 623-7.

7. Colombini A., Corsetti R., Machado M., et al. (2012). Serum creatine kinase activity and its relationship with renal function indices in professional cyclists during the Giro d'Italia 3-week stage race. Clin J Sport Med, 22(5), 408-13.

8. Gocentas A., Landõr A. (2012). Stress and muscle damage monitoring in highlevel basketball players. Papers on Anthropology, 21, 72-83.

9. Gorce-Dupuy A. M., Vela C., Badiou S., et al. (2012). Antioxidant and oligonutrient status, distribution of amino acids, muscle damage, inflammation, and evaluation of renal function in elite rugby players. Clin Chem Lab Med, 50, 1777-89.

10. Hoffman M. D., Stuempfle K. J., Fogard K., et al. (2013). Urine dipstick analysis for identification of runners susceptible to acute kidney injury following an ultramarathon. J Sports Sci, 31(1), 20-31.

11. Lazarim F. L., Antunes-Neto J. M., da Silva F. O., et al. (2009). The upper values of plasma creatine kinase of professional soccer players during the Brazilian National Championship. J Sci Med Sport, 12(1), 85-90.

12. Levey A. S., Coresh J., Greene T., et al., Chronic Kidney Disease Epidemiology Collaboration (2006). Using standardized serum creatinine values in the modification of diet in renal disease study equation for estimating glomerular filtration rate. Ann Intern Med, 145, 247-54.

13. Lippi G., Banfi G., Luca Salvagno G., et al. (2008). Comparison of creatinine-based estimations of glomerular filtration rate in endurance athletes at rest. Clin Chem Lab Med, 46, 235-9.

14. Lugaresi R., Leme M., de Salles Painelli V., et al. (2013). Does long-term creatine supplementation impair kidney function in resistance-trained individuals consuming a high-protein diet? J Int Soc Sports Nutr, 10(1), 26.

15. Machado M., Zini E. N., Valadão S. D., et al. (2012). Relationship of glomerular filtration rate and serum CK activity after resistance exercise in women. Int Urol Nephrol, 44(2), 515-21.

16. Manzi V., D’Ottavio S., Impellizzeri F. M., et al. (2010). Profile of weekly training load in elite male professional basketball players. J Strength Cond Res, 24(5), 1399-406.

17. McLellan C. P., Lovell D. I., Gass G. C. (2011). Markers of postmatch fatigue in professional Rugby League players. J Strength Cond Res, 25(4), 1030-9.

18. Meeusen R., Duclos M., Foster C., et al. (2013). European College of Sport Science; American College of Sports Medicine. Prevention, diagnosis, and treatment of the overtraining syndrome: joint consensus statement of the European College 
of Sport Science and the American College of Sports Medicine. Med Sci Sports Exerc, 45(1), 186-205.

19. Meyer T., Meister S. (2011). Routine blood parameters in elite soccer players. Int J Sports Med, 32(11), 875-81.

20. Milić R., Banfi G., Del Fabbro M., et al. (2011). Serum creatinine concentrations in male and female elite swimmers. Correlation with body mass index and evaluation of estimated glomerular filtration rate. Clin Chem Lab Med, 49, 285-9.

21. Reinke S., Karhausen T., Doehner W., et al. (2009). The Influence of Recovery and Training Phases on Body Composition, Peripheral Vascular Function and Immune System of Professional Soccer Players. PLoS ONE 4(3): e4910. doi:10.1371/journal.pone.0004910

Address for correspondence:

Anatoli Landõr

University of Tartu

Faculty of Medicine

Ravila 19, Tartu, 50406, Estonia

E-mail: anatolilandor@gmail.com 